\title{
ADAM10 and ADAM17 in Liver Regeneration and Fibrosis: Regulation of EGFR, c-Met and TNF RI Signalling
}

Olga Zbodakova ( $\nabla$ olga.zbodakova@img.cas.cz )

Institute of Molecular Genetics

Karel Chalupsky

Czech Centre of Phenogenomics, Institute of Molecular Genetics

Lenka Sarnova

Institute of Molecular Genetics

Petr Kasparek

Institute of Molecular Genetics

Marketa Jirouskova

Institute of Molecular Genetics

Martin Gregor

Institute of Molecular Genetics

Radislav Sedlacek

Czech Centre of Phenogenomics, Institute of Molecular Genetics

\section{Research Article}

Keywords: TACE, shedding, Met, growth factor, TNFR1

Posted Date: February 11th, 2021

DOl: https://doi.org/10.21203/rs.3.rs-184254/v1

License: (9) (i) This work is licensed under a Creative Commons Attribution 4.0 International License.

Read Full License 


\section{Abstract}

ADAM10 and ADAM17 are proteases that affect multiple signalling pathways by releasing molecules from the cell surface. As their substrate specificities partially overlaps, we investigated their concurrent role in liver regeneration and fibrosis, using three liver-specific deficient mouse lines: ADAM10- and ADAM17-deficient lines, and a line deficient for both proteases. In the model of partial hepatectomy, double deficient mice exhibited decreased AKT phosphorylation, decreased release of EGFR activating factors and lower shedding of HGF receptor c-Met. Thus, simultaneous ablation of ADAM10 and ADAM17 resulted in impaired EGFR signalling, while HGF/C-Met signalling pathway was enhanced. In contrast, antagonistic effects of ADAM10 and ADAM17 were observed in the model of chronic $\mathrm{CCl}_{4}$ intoxication. While ADAM10-deficient mice develop more severe fibrosis manifested by high ALT, AST, ALP and higher collagen deposition, combined deficiency of ADAM10 and ADAM17 surprisingly results in comparable degree of liver damage as in control littermates. Therefore, ADAM17 deficiency is not protective in fibrosis development per se, but can ameliorate the damaging effect of ADAM10 deficiency on liver fibrosis development and results in decreased shedding of TNF RI, while ADAM10 deficiency leads to increased levels of soluble TNF RI in serum. In conclusion, hepatocyte-derived ADAM10 and ADAM17 are important regulators of growth receptor signalling and TNF RI release, and pathological roles of these proteases are dependent on the cellular context.

\section{Introduction}

The liver is an organ with remarkable regenerative capacity. Yet, once this ability is compromised, liver transplantation often remains the only solution. Therefore, studying mechanisms contributing to the maintenance of its homeostasis is very important.

A well tolerated and widely used rodent model of liver regeneration is $2 / 3$ partial hepatectomy [1]. In this model, two thirds of the liver are excised which induces hepatocyte proliferation in the remaining tissue to compensate for the original liver mass [2]. In the priming phase, the initial phase of regeneration after partial hepatectomy, differentiated $G_{0}$ hepatocytes are stimulated by cytokines and re-enter cell cycle. Previous studies have shown that this inceptive part of healing process is governed to a large extent by two cytokines, tumor necrosis factor alpha (TNF-a) and interleukin $6($ IL-6) $[3,4]$. In the following phase, in which hepatocytes proceed through cell cycle, two growth receptor pathways were shown to play a crucial part: epidermal growth factor receptor (EGFR) [5] [6] and hepatocyte growth factor (HGF) receptor (c-Met) pathway [7-9]. Combined elimination of both EGFR and HGF/c-Met pathways fatally disrupted homeostasis in an unchallenged liver [10] and completely hindered liver regeneration after partial hepatectomy [11].

EGFR can be activated by different ligands: epidermal growth factor (EGF), transforming growth factor a (TGFa), amphiregulin (AREG), betacellulin, heparin-binding EGF-like growth factor (HB-EGF), epiregulin and epigen [12]. In the liver, a major source of AREG and TGFa are the hepatocytes themselves [13], HBEGF is predominantly produced by a non-parenchymal liver cells [14], and EGF is supplied to the liver 
from intestinal cells [15]. Especially, HB-EGFR [16] and AREG [17] were reported as a potent stimulators of hepatocyte proliferation. In contrast to the EGFR pathway, the signalling pathway downstream of the cMet receptor is activated exclusively by $\operatorname{HGF}[18,19]$, which is mainly produced by a non-parenchymal liver cells [19]. Ligand binding leads to phosphorylation of growth receptors and activation of downstream mediators (such as ERK1/2, STAT3 and AKT) [2].

Several of the mentioned protein factors critical for liver regeneration are released from the cell surface by proteases. The most important of these are the membrane-bound zinc-binding proteases ADAM17 and ADAM10, whose activation leads to substrate cleavage proximal to the cell surface [20], in the process called ectodomain 'shedding'. The importance of ADAMs is highlighted by the fact that both, ADAM10[21] and ADAM17- [22] deficient mice are not viable and die either prenatally or shortly (ADAM10) after birth (ADAM17). Observed lethal developmental defects were attributed either to insufficient Notch signalling (ADAM10-deficient mice) [21] or to aberrant EGFR signalling (ADAM17 deficiency) [22]. Although liver development was not compromised in ADAM10- or ADAM17-deficient animals, ADAM10 seems to be important regulator for liver homeostasis maintenance as liver-specific ablation of ADAM10 resulted in spontaneous fibrogenesis, characterized by unbalanced differentiation of hepatocytes and deregulated expression of the bile acid transporters [23].

ADAM17 is an important regulator of TNF-a pathway. Besides cleavage of TNF-a itself, which is necessary in order to bind and activate its receptor, ADAM17 also sheds TNF receptor 1 (TNF RI) and TNF receptor 2 (TNF RII) [24], which leads to pathway inactivation. ADAM17 activity thus regulates TNF-a signalling in two directions and the outcome of ADAM17 activity on TNFa signalling therefore depends on the cellular context. Both liver macrophages and hepatocytes are a prominent source of TNF-a during liver injury [25] [26]. EGFR pathway is regulated by ADAMs[27] [28] through the release of EGFR ligands. Surprisingly, liver-specific ADAM17-deficient mice did not exhibit delayed hepatocyte proliferation after partial hepatectomy, even though levels of AREG were significantly reduced in these mice [25]. Similar to the EGFR pathway, HGF signalling is also regulated via the shedding process. Several studies have shown that the HGF receptor c-Met is cleaved by both ADAM10 and ADAM17 [29] [30].

In conclusion, ADAM10 and ADAM17 are implicated in several pathways crucial for liver regeneration. In this work, we aimed to examine the combined role of ADAM10 and ADAM17 in liver regeneration and liver fibrosis development, using mouse models deficient in ADAM10, ADAM17 or simultaneously deficient for both proteases.

\section{Results}

\section{Generation of liver-specific ADAM10- and ADAM17- deficient mouse models}

To study the role of ADAM10 and ADAM17 in hepatic injury, we generated three liver-specific deficient lines, using a mouse line expressing Cre under the human albumin promoter. Besides single deficient 
ADAM10 $\triangle 0^{\triangle \mathrm{Alb}}$ and $A D A M 17^{\triangle \mathrm{Alb}}$ lines, we also generated a double deficient $A D A M 10^{\triangle \mathrm{Alb}} \mathrm{ADAM} 17^{\triangle \mathrm{Alb}}$ line as both proteases share several substrates, which has been previously described as important in liver pathology. The ablation of Adam 10 and $A d a m 17$ genes was verified by semiquantitative PCR using genomic DNA isolated from primary hepatocytes (Fig. 1a). Furthermore, lack of Adam10 and Adam17 expression was confirmed by quantitative PCR using RNA isolated from whole liver tissue (Fig. 1b).

Previously Muller et al.[23] reported that ADAM10-deficient mice, which had stronger expression of Cre driven by a-fetoprotein promotor, but same tissue specificity as our ADAM10 $\triangle \mathrm{Alb}$, develop spontaneous fibrosis. Interestingly, in our experimental setup low serum activity of alanine aminotransferase (ALT) and aspartate aminotransferase (AST), which are the markers of hepatocellular damage, indicated that neither $A D A M 10^{\triangle A l b}$, nor ADAM17 $\triangle \mathrm{Alb}, A D A M 10^{\triangle \mathrm{Alb}} \mathrm{ADAM} 17^{\triangle \mathrm{Alb}}$ mice developed spontaneous liver damage (Fig. 1C). Furthermore, the gross morphology of liver did not display any abnormalities in any of the mutant lines.

\section{ADAM10 ${ }^{\triangle A l b}$ ADAM17 $\triangle \mathrm{Alb}$ mice have reduced AKT activation after partial hepatectomy}

As unchallenged animals (ADAM10 $\left.{ }^{\triangle A l b}, A D A M 17^{\triangle A l b}, A D A M 10^{\triangle A l b} A D A M 17^{\triangle A l b}\right)$ did not display any significant alteration in markers of liver damage when compared to their control littermates (Ctrl) littermates, we tested whether ADAM10/17 deficiency had an effect on model liver pathologies. First, we examined the regenerative ability of the liver by applying the model of $2 / 3$ partial hepatectomy. The typical pattern of elevated levels of cytokine IL-6, corresponding to activation of resident liver macrophages, was observed in the serum of all treated animals three hours post hepatectomy with no difference between ADAM10 $10^{\triangle \mathrm{Alb}}, \mathrm{ADAM} 7^{\triangle \mathrm{Alb}}, \mathrm{ADAM} 0^{\triangle \mathrm{Alb}} \mathrm{ADAM} 17^{\triangle \mathrm{Alb}}$ mice and Ctrl (Fig. 2a). As ADAM10/17-dependent release of c-Met into serum has been previously described to be associated with liver damage [30], we monitored levels of soluble c-Met in our model. We found that c-Met serum levels are elevated at 3 hours post hepatectomy in all lines irrespective of genotype. These levels remained significantly above unchallenged levels at all studied time points $(3,6,40$ and 72 hours post hepatectomy, Fig. $3 \mathrm{~d}$ ). ADAM10 ${ }^{\triangle \mathrm{All}}$ and $\mathrm{ADAM} 17^{\triangle \mathrm{Alb}}$ mice exhibited c-Met serum levels comparable to Ctrl littermates 3 hours post hepatectomy. However, shedding of c-Met was significantly inhibited in $A D A M 10^{\triangle A l b} A D A M 17^{\triangle A l b}$ mice in comparison with Ctrl or single deficient lines (Fig. 2a).

To study the activation of pro-survival signals following the initial priming, we analysed the activation of AKT kinase, which plays a crucial role in proliferation of hepatocytes [31]. Immunoblot analysis of whole liver lysates of hepatectomized mice revealed comparable levels of AKT phosphorylation in ADAM10 $\triangle A l b$ and ADAM17 $\triangle \mathrm{Alb}$ mice compared to Ctrl 6 hours post hepatectomy (Fig. 2b-c). In contrast, double deficient $A D A M 10^{\triangle A l b} A D A M 17^{\triangle A l b}$ mice exhibited significantly reduced levels of phosphorylated $A K T$ at this time point. This suggests that both ADAM10 and ADAM17 contribute to the release of factors which trigger proliferative signals after partial hepatectomy and that they are interchangeable in activation of AKT pathway. 


\section{Reduced amounts of EGFR activating factors in serum of ADAM10 ${ }^{\triangle A l b} A D A M 17^{\triangle A l b}$ mice 6 hours after partial hepatectomy}

As the EGFR pathway is a well-established target of both ADAM10 and ADAM17, we next analysed whether reduced AKT activation might result from aberrant EGFR signalling. Therefore, we first checked the extent of release of AREG, a well-known EGFR ligand, in primary ADAM10 $\triangle \mathrm{Alb} A D A M 17^{\triangle \mathrm{Alb}}$ hepatocyte cultures. AREG levels in culture media were measured by ELISA at 6 hours in cultures of untreated ADAM10 ${ }^{\triangle \mathrm{Alb}} \mathrm{ADAM} 17^{\triangle \mathrm{Alb}}$ and Ctrl hepatocytes in the presence of $10 \% \mathrm{FBS}$. ADAM10 $\mathrm{Allb} A D A M 17^{\triangle \mathrm{Alb}}$ primary hepatocytes exhibited a dramatically reduced ability to effectively shed AREG (Fig. 2d).

Compromised AREG shedding in ADAM10 $\triangle \mathrm{Alb} A D A M 17^{\triangle \mathrm{Alb}}$ hepatocytes prompted us to check the release of EGFR ligands in vivo - in serum from peripheral blood of mice after partial hepatectomy. To evaluate the effectiveness of EGFR ligand shedding, we incubated Ctrl hepatocytes in the presence of serum obtained from $A D A M 10^{\triangle A l b} A D A M 17^{\triangle A l b}$ mice or $C t r l 6$ hours post hepatectomy for 30 minutes and determined its ability to trigger EGFR phosphorylation. Immunoblot analysis revealed that ADAM10 ${ }^{\triangle A l b} A D A M 17^{\triangle A l b}$ triggered significantly lower phosphorylation of EGFR than serum from Ctrl (Fig. 2e-f). This result strongly suggests that the shedding of EGFR ligands is compromised in

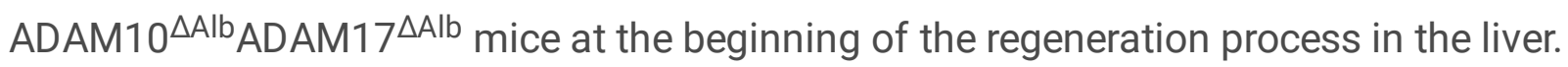

\section{ADAM10 ${ }^{\Delta \text { Alb }}$ ADAM17 ${ }^{\Delta \text { Alb }}$ hepatocytes show no defect in proliferation in response to partial hepatectomy}

Lower levels of AKT phosphorylation in ADAM10 ${ }^{\triangle A l b} A D A M 17^{\triangle A l b}$ at early time points after hepatectomy suggested a slower proliferative response; therefore we next examined hepatocyte proliferation in the following stages of liver regeneration.

To follow liver mass restoration, we monitored changes in liver weight to body weight ratio in ADAM $10^{\triangle A \mid b}$ ADAM $17^{\triangle A l b}$ mice consecutively three days after hepatectomy. In this time frame, ADAM $10^{\triangle \mathrm{Alb}} \mathrm{ADAM} 17^{\triangle \mathrm{Alb}}$ mice gained liver mass to comparable extent as Ctrls, reaching increases of 50 $\%$ within 72 hours (Fig. 3a). To examine whether the described increase reflected the rate of hepatocyte division, we next analysed hepatocytes proliferation using immunohistological staining of proliferating cell nuclear antigen (PCNA) (Fig. 3b). For quantification, only nuclei corresponding to hepatocytes, identified by their typical round shape, were counted, while all non-parenchymal cells were excluded from the analysis. Surprisingly, no difference in number of PCNA positive nuclei was detected between ADAM $10^{\triangle A l b} A D A M 17^{\triangle A l b}$ and $\mathrm{Ctrl}$ mice at 40 hours post hepatectomy (Fig. 3b). This suggests that despite the initial decrease in the activation of EGFR pathway, ADAM $10^{\triangle A l b} A D A M 17^{\triangle A l b}$ mice are able to proceed with hepatocyte proliferation as effectively as Ctrls. 


\section{HGF triggers stronger signalling through cMet in ADAM10 ${ }^{\Delta A l b} A D A M 17^{\triangle A l b}$ hepatocytes}

To explore the ability of $A D A M 10^{\triangle A l b} A D A M 17^{\triangle A l b}$ to compensate for lower EGFR activation, we examined other signalling pathways important for hepatocyte proliferation. The HGF/c-Met pathway was a prospective candidate, as it plays an important role in liver regeneration and c-Met is a substrate of both ADAM10 and ADAM17. To find out whether HGF/c-Met pathway is dysregulated in ADAM $10^{\triangle A l b} A D A M 17^{\triangle A l b}$ mice in the model of partial hepatectomy, we next analysed serum levels of both HGF and the soluble form of the HGF receptor c-Met. ELISA analysis revealed a prominent increase of serum HGF levels mice 40 hours post hepatectomy, however no significant difference was observed in any of the studied mouse lines, ADAM10 $\triangle \mathrm{Alb}, A D A M 17^{\triangle \mathrm{Alb}}, \mathrm{ADAM} 10^{\triangle \mathrm{Alb}} \mathrm{ADAM} 17^{\triangle \mathrm{Alb}}$ compared to their Ctrl littermates (Fig. 3c, S1). Together with increased levels of HGF, levels of c-Met were significantly elevated in Ctrl animals as well as in ADAM10 $\triangle \mathrm{Alb}$ and ADAM17 $\triangle \mathrm{Alb}$ lines (Fig. 2a, S1). However, we found that the level of soluble c-Met in serum of $A D A M 10^{\triangle A l b} A D A M 17^{\triangle A l b}$ was significantly reduced in comparison to Ctrls at all studied time points (Fig. 3d, S1).

Reduced c-Met levels in serum correspond to compromised shedding in ADAM10 ${ }^{\triangle A l b} A D A M 17^{\triangle A l b}$ mouse and may imply a higher level of remaining functional c-Met on hepatocyte surface leading to amplified HGF signalling. To address this hypothesis in vitro, we isolated primary hepatocytes from ADAM10 ${ }^{\triangle \mathrm{Alb}} \mathrm{ADAM} 17^{\triangle \mathrm{Alb}}$ and Ctrl mice and treated them with HGF in serum free medium. Indeed, immunoblot analysis of hepatocyte lysates revealed higher levels of phosphorylation of both c-Met and AKT (Fig. 3e-f) in ADAM10 ${ }^{\triangle \mathrm{Alb}} \mathrm{ADAM} 17^{\triangle \mathrm{Alb}}$ compared to Ctrl cells upon HGF treatment. Thus, combined deficiency of ADAM10 and ADAM17 results in reduced shedding of c-Met, retention of a larger pool of unshed receptor on the hepatocyte surface and higher sensibility towards HGF.

\section{ADAM10 ${ }^{\Delta A l b}$ mice exhibit higher susceptibility to $\mathrm{CCl}_{4}$-induced fibrosis}

As we identified alterations in signalling pathways during liver regeneration in $A D A M 10^{\triangle A l b} A D A M 17 \triangle A l b$ mice, we were further interested how the deficiency of ADAM10 and ADAM17 may affect the development of liver injury. We therefore applied a model of 4-week $\mathrm{CCl}_{4}$-intoxication, which results in liver fibrosis.

As expected, markers of liver injury (serum activity of alanine aminotransferase (ALT) and aspartate aminotransferase (AST)), were elevated in all studied groups after 4 weeks of $\mathrm{CCl}_{4}$ treatment. Strikingly the highest hepatocellular damage was found in ADAM10 $\triangle \mathrm{Alb}$ animals (Fig. 4a-b), while ADAM17 $\triangle \mathrm{Alb}$ mice exhibited the same extent of damage as their Ctrl littermates. Interestingly, serum aminotransferases were not increased in $\mathrm{ADAM}_{10}{ }^{\triangle \mathrm{Alb}} \mathrm{ADAM} 17^{\triangle \mathrm{Alb}}$ in contrast to single ADAM10 $10^{\triangle \mathrm{Alb}}$ mice. We further evaluated the development of fibrosis in liver by histopathological staining of fibrillary collagens, where the Sirius Red positive (SR+) area was determined by image analysis. Our results 
showed that higher levels of serum liver damage markers correlated with most extensive deposition of fibrillary collagens in ADAM10 $\triangle \mathrm{Alb}$ mice when compared to other deficient lines and Ctrls (Fig. 4c). Sirius Red staining (Fig. 4d) showed that at this time point ADAM10 $\triangle \mathrm{Alb}$ developed a more advanced bridging phase of fibrosis, in which collagen scars already interconnected central veins. The extent of collagen deposits of $A D A M 17^{\triangle A l b}$ and also ADAM $10^{\triangle A l b} A D A M 17^{\triangle A l b}$ were comparable to Ctrls. Fibrotic scars in these lines stretched from the central vein but were not prominent enough to form bridges. Moreover, ki67 staining revealed significantly higher hepatocyte proliferation (Fig. 4e-f) in the ADAM10 $\triangle$ Alb mouse line, which is most likely caused by a proliferative response to more severe damage caused by $\mathrm{CCl}_{4}$.

A prior study [23] has shown that ADAM10 downregulation decreased expression of several bile acid transporters, including Mrp2. As other members of Mrp family, Mrp3 and Mrp4, are involved in detoxification of $\mathrm{CCl}_{4}$, we analysed their expression at the mRNA level in our model using qPCR. In contrast, in our model ADAM10 $\triangle \mathrm{Alb}$ mice displayed no significant differences in Mrp2 liver expression after 4-weeks of $\mathrm{CCl}_{4}$-intoxication, as well as in Mrp3 and Mrp4 (S2). Hence, the higher susceptibility of ADAM $10^{\triangle \mathrm{Alb}}$ towards $\mathrm{CCl}_{4}$ treatment is not a consequence of the difference in transporter proteins expression.

\section{ADAM10 ${ }^{\Delta A l b}$ biliary epithelial cells are affected after $\mathrm{CCl}_{4}$ intoxication}

Serum analysis revealed that $A D A M 10^{\triangle A l b}$ mice exhibit not only higher levels of hepatocellular damage markers (ALT and AST), but also markers of biliary damage, such as alkaline phosphatase (ALP). ALP activity was elevated in the serum of $A D A M 10^{\triangle A l b}$ mice after 4 weeks of $\mathrm{CCl}_{4}$ treatment in comparison with Ctrl, but not in ADAM17 $\triangle \mathrm{Alb}$ or ADAM10 ${ }^{\triangle \mathrm{Alb}} \mathrm{ADAM} 17^{\triangle \mathrm{Alb}}$ (Fig. 5a). To determine whether elevated ALP levels are connected to hyperproliferation of biliary epithelia, we stained liver sections with CK19, a marker of biliary epithelial cells. ADAM10 $\triangle \mathrm{Alb}$ livers did not differ in the CK19 positive (CK19+) area from Ctrls, and neither did ADAM17 $\triangle \mathrm{Alb}$ or $\mathrm{ADAM} 0^{\triangle \mathrm{Alb}} \mathrm{ADAM} 17^{\triangle \mathrm{Alb}}$ (Fig. 5b). On the other hand, higher levels of CD44 protein, a molecule overexpressed in proliferating biliary epithelium and a substrate of ADAM10, were detected in lysates from ADAM10 $\triangle$ Alb livers than in Ctrls (Fig. $5 \mathrm{C}$ ). A higher amount of CD 44 was not determined in lysates from ADAM10 $\triangle \mathrm{Alb} A D A M 17^{\triangle \mathrm{Alb}}$ livers. The observed activation of biliary epithelial cells can be caused by a higher degree of overall liver damage, caused by chronic intoxication in ADAM10 ${ }^{\triangle A l b}$ mice. Importantly, ADAM17 deficiency overcomes the biliary damage enhanced by ablation of ADAM10.

\section{Shedding of TNF RI is affected by deficiency of ADAM17 and ADAM10}

Previous studies have shown that $\mathrm{CCl}_{4}$-induced liver damage is connected with elevated levels of soluble TNF RI (sTNF RI) and TNF RII [32]. As TNF receptors are well-described substrates of ADAM17, we were interested how ablation of ADAM17 and ADAM10 in hepatocytes affects levels of sTNF RI in our models. 
In consistency with previous reports, we observed an increase of sTNF RI serum levels after 4 weeks of $\mathrm{CCl}_{4}$ treatment in all studied groups in comparison to untreated animals. As expected, ADAM17 $\triangle \mathrm{Alb}$ displayed significantly lower serum levels of sTNF RI than Ctrl littermates (Fig. 6a). Interestingly, ADAM10 ${ }^{\triangle \mathrm{Alb}}$ ADAM17 $\triangle \mathrm{Alb}$ mice did not show significant differences to Ctrls. Surprisingly, ADAM10 $\triangle \mathrm{Alb}$ exhibited the opposite situation with higher levels of TNFRI in serum than Ctrl littermates (Fig. 6a). Increase of sTNF RI was found also in the serum of unchallenged ADAM10 $\triangle \mathrm{Alb}$ animals $(\mathrm{Ctrl}=740 \mathrm{pg} / \mathrm{ml}$, $\left.\mathrm{ADAM} 10^{\triangle \mathrm{Alb}}=1165 \mathrm{pg} / \mathrm{ml}, \mathrm{p}=0.0012\right)$, as well as ADAM10 $\triangle \mathrm{Alb}$ primary hepatocytes without any challenge (Fig. 6b). To exclude that identified differences originated from a changed TNF RI expression, we performed qPCR using RNA from whole liver tissue. No differences in Tnfr1 transcript were found between individual mouse lines (Fig. 6c), indicating that observed differences resulted from alteration in sTNF RI release.

While ADAM17 $\triangle$ Alb mice exhibit reduced shedding of sTNF RI and ADAM10 $\triangle \mathrm{Alb}$ mice exhibit an increased release of sTNF RI into serum, the situation in $A D A M 10^{\triangle A l b} A D A M 17^{\triangle A l b}$ was dependent on the type of the liver challenge. Under physiological conditions, we determined higher levels of sTNF RI ADAM10 ${ }^{\triangle \mathrm{Alb}}$ ADAM17 $\triangle \mathrm{Allb}$ compared to Ctrl (Fig. 6d), similar to ADAM10 ${ }^{\triangle \mathrm{Alb}}$. However, after partial hepatectomy, $A D A M 10^{\triangle \mathrm{Alb}} A D A M 17^{\triangle \mathrm{Alb}}$ resembled $A D A M 17^{\triangle \mathrm{Alb}}$ with hampered release of sTNF RI (Fig. 6d). ADAM10 ${ }^{\triangle A l b} A D A M 17^{\triangle A l b}$ primary hepatocytes exhibit inhibited shedding of TNF RI, similarly to $A D A M 17^{\triangle A l b}$. As isolation of primary hepatocytes is linked to their activation due to the treatment with digestive enzymes, we can consider their state closer to the conditions in hepatectomized rather than unchallenged liver. A regulatory role of sTNF RI in liver pathological states is emerging [33] [34] [35]. Our data suggest that both ADAM10 and ADAM17 influence its release.

\section{Discussion}

ADAM10 and 17 are metalloproteinases that modulate signalling in many physiological processes. For the liver regeneration, EGFR and c-Met pathways were identified as the most important ones. Several recent articles showed that only concurrent inactivation of both pathways causes inability of liver to recover from the partial hepatectomy and higher necrosis after $\mathrm{CCl}_{4}$ intoxication [11] [36] [7]. In this study, we demonstrate that both pathways are modulated by ADAM proteases in a pathological liver. Simultaneous hepatocyte-specific deficiency of both ADAM10 and ADAM17 led to lower activation of EGFR as well as to the lower shedding of a c-Met receptor. Thus, initial slower regenerative response after partial hepatectomy, caused by impaired shedding of EGFR activating ligands, was compensated by stronger HGF signalling through c-Met receptor. Furthermore we show, that both proteases are also involved in TNF RI release. ADAM17 directly cleaves the receptor, while ADAM10 deficiency causes elevation of TNF RI serum levels through an unidentified process.

Several EGFR ligands promote the proliferation of hepatocytes. It has been described that expression of HB-EGF, TGF-a and AREG is elevated after partial hepatectomy, even though they are not completely fungible [37]. ADAM17 has been described as the main protease in the release of TGF-a and AREG [27]. 
HB-EGF is shed mainly by ADAM17 and ADAM12, and betacellulin is a substrate of ADAM10 [27]. In addition, EGF [38] supplied to the liver from the duodenum, was identified as a substrate of ADAM10 [39]. We show that combined deletion of ADAM10 and ADAM17 in hepatocytes leads to lower levels of EGFR activating factors in the serum from peripheral blood after 2/3 partial hepatectomy. This was demonstrated by lower levels of phosphorylated AKT in ADAM10 ${ }^{\triangle A l b} A D A M 17 \triangle A l b$ mice 6 hours post hepatectomy, which was not observed in single deficient mice. Moreover, decreased phosphorylation of EGFR was observed in the cultured cells treated by mouse serum originating from ADAM $10^{\triangle A l b} A D A M 17^{\triangle A l b}$ mice after hepatectomy. Altogether this indicates that hepatocytes alone are a significant source of growth factors which modulate their cell cycle. Similar results were described by McMahan et al. [33] in hepatocyte-specific ADAM17-deficient mice, in which dramatically reduced levels of AREG were observed. However, AREG levels in their study were measured in liver tissue lysates and therefore represented the expression of a protein, not its shed pool. In our model we did not observe differences of expression in AREG protein but we clearly showed that the deletion of ADAM proteases influenced release of EGFR ligands to the serum. Our data indicate that not only ADAM17, but also ADAM10 is a regulator of EGFR ligand shedding after partial hepatectomy. Of note is that factors released from hepatocytes can potentially influence also other liver cell types, e.g. hepatic stellate cells. This may have a negative impact in conditions such as liver fibrosis when enhanced HSC proliferation is not desired.

Similarly to EGFR, HGF signalling through c-Met receptor is another independent pathway leading to the proliferation of hepatocytes [7] [40]. In our model, serum HGF was strongly increased after partial hepatectomy. On the other hand, we showed that shedding of c-Met was also elevated, especially in the first hours after the hepatectomy, which is probably part of a regulatory mechanism of HGF signalling. We observed that isolated primary ADAM10 and ADAM17 double-deficient hepatocytes had a more prominent response to HGF treatment. We concluded that these differences in HGF response were caused by higher availability of c-Met receptor on cell surface due to its limited shedding in the combined absence of ADAM10 and ADAM17. Shedding of c-Met did not significantly differ in the absence of ADAM10 or ADAM17 individually. The question remains whether shedding of the c-Met receptor results only in lower availability of functional receptor on the cell surface, or if there is an additional contribution of a solubilized part of the receptor. Released cleft fragment of c-Met could bind HGF and prevent binding of HGF to functional surface-bound receptors. We did not identify this decoy function of a c-Met fragment in our in vitro conditions, but further investigation is desired.

In summary, the deletion of ADAM10 and ADAM17 driven by the albumin promoter leads to hampered EGFR signalling, but enhanced HGF/c-Met signalling in regenerating hepatocytes. As a result, ADAM $10^{\triangle A l b} A D A M 17 \triangle A l b$ mice did not show any alteration from Ctrl littermates 40 hours post hepatectomy, hepatocytes proceeded towards proliferation and liver weights increased normally.

We have reported previously that liver-specific ADAM10-deficient mice, with stronger expression of Cre (Alb-Cre homozygotes or a-fetoprotein-Cre hemizygotes), developed spontaneous fibrosis [23]. This was not the case in mice used in this study (Alb-Cre hemizygotes). ADAM10 $\triangle \mathrm{Alb}$ mice were monitored up to 33 
weeks of age, and none of the fibrotic markers were increased in unchallenged animals. The lower expression levels of Cre in hemizygote animals resulted in sustained expression of ADAM10 in a negligible proportion of hepatocytes, which was sufficient to protect from development of spontaneous fibrosis. However, ADAM10 ${ }^{\triangle A l b}$ mice were much more susceptible to liver damage than control littermates when exposed to the toxic agent. Difference was not apparent after acute intoxication (single dose of $\mathrm{CCl}_{4}$ ) but was clearly distinct after 4 weeks of chronic $\mathrm{CCl}_{4}$ treatment. The reason for the higher susceptibility of ADAM10-deficient mice towards $\mathrm{CCl}_{4}$ intoxication is still not clear. It was shown that ADAM10 directly influences the expression of bile acid transporters, e.g. MRP2 [23], which play an important role in removal of toxic products generated in hepatocytes after $\mathrm{CCl}_{4}$ treatment. However, we did not find the difference in Mrp2 mRNA expression, nor in the expression of Mrp3 and Mrp4 which are involved in $\mathrm{CCl}_{4}$ detoxication [41]. The difference in $\mathrm{Mrp} 2$ expression between two studies may have arisen due to the differing nature of the injuries evoked in the studies, as we used toxin- induced injury, while model of Muller et al. [23] resulted from an inner imbalance of liver homeostasis.

Not only levels of ALT and AST were increased in ADAM10 ${ }^{\triangle A l b}$, but also ALP. ALP in liver damage is connected with activated biliary epithelial cell. This is in line with previously published data which suggest that ADAM10-deficient mice have increased ductular reaction [23]. Consistently with this, we found that $A D A M 10^{\triangle A l b}$ had increased protein levels of CD44 in liver lysates. Biliary epithelial cell are a prominent source of CD44 in liver [42], and its expression is connected to the proliferation of biliary epithelia. The identified increase of CD44 levels could be directly linked to ADAM10 deficiency, as ADAM10 is the primary sheddase for CD44 [43].

Interestingly, the hepatocyte damage in $A D A M 10^{\triangle A l b} A D A M 17^{\triangle \mathrm{Alb}}$ and $A D A M 17^{\triangle \mathrm{Alb}}$ mice was comparable to $\mathrm{Ctrl}$ littermates. Thus, ADAM17 elimination was not protective in $\mathrm{CCl}_{4}$ induced liver fibrosis when functional ADAM10 was present, but alleviated aggravating impact of ADAM10 deficiency on fibrosis development. Similarly, we have shown previously [44] that ADAM17 inhibition by ursodeoxycholic acid is protective in model of cholestatic injury. In contrast, recent work from Sundaram et al. [33], showed that impaired ADAM17 maturation exacerbated bile duct obstruction induced fibrosis. The main difference between their work and our present findings is the cell type affected by genetic models. Our model leads to specific ablation of ADAM17 in hepatocytes and biliary epithelial cells. Sundaram et al. studied mice with whole body deficiency of iRhom 2 that leads to the inhibition of ADAM17 in cell types, in which iRhom 2 governs maturation of ADAM17. They explained their phenotype through the suppression of ADAM17 in hepatic stellate cells, a cell type which was not affected in our genetic model. This shows that the modulation of ADAM17 activity on different cell types within the liver can lead to opposite pathological consequences.

In summary, ADAM $10^{\triangle A l b}$ are more prone to chronic intoxication, but subsequent proliferation of hepatocytes is not affected. Interestingly, ADAM17 deficiency counteracted the negative effect of ADAM10 deficiency in liver fibrosis development. ADAM10 ${ }^{\triangle A l b} A D A M 17^{\triangle A l b}$ animals were the same as Ctrl 
littermates in all studied parameters during fibrosis development. Further deciphering of molecular mechanism behind this effect represent interesting area for future research.

Role of ADAM17 in TNF-a shedding is very well-known. However, growing number of evidence suggest the importance of TNF receptors shedding in the regulation of TNF-a signalling [32]. Shedding of TNF RI or TNF RII not only disables functionality of this receptor on the cell surface but released part - soluble receptor, can bind and limit the availability of TNF- a. Role of TNF RI in liver injury is not easy to interpret. Inhibition of TNF RI led to lower hepatocellular damage after $\mathrm{CCl}_{4}$ treatment [45]. On the other hand, the different report described the importance of TNF RI or liver regeneration after $\mathrm{CCl}_{4}$ intoxication, as mice lacking TNF RI showed inhibited hepatocyte DNA synthesis [46]. A recent study showed that TNF RI deficiency led to the increased cholestatic injury in the model of chronic hepatitis, mediated through higher infiltration of immune cells [47]. In our study, we found that ADAM17 deficiency in hepatocytes leads to lower levels of TNFRI in serum in both studied liver challenges, as expected. Surprisingly, ADAM10-deficient animals consistently show higher levels of TNF RI in serum than their Ctrl littermates. Same results were confirmed in vitro on primary hepatocytes, suggesting elevated serum levels of sTNF $\mathrm{RI}$ in vivo had a source in ADAM10-deficient hepatocytes. This effect is most likely not caused by compensational activation of ADAM17, as other substrates of ADAM17, TNF- $a$ and AREG, were not elevated in the medium of ADAM10-deficient cells. Moreover, ADAM $10^{\triangle A l b} A D A M 17^{\triangle A l b}$ mice show an interesting pattern in levels of TNF RI in serum. In an unchallenged state, ADAM $10^{\triangle A l b} A D A M 17^{\triangle A l b}$ exhibit increased shedding of TNF RI similarly to $A D A M 10^{\triangle A l b}$, while during the liver challenge, double deficient mice were comparable to ADAM17 $\triangle \mathrm{Alb}$ or Ctrl littermates. This nicely shows that the activity of ADAM17 is induced in liver pathological states and the lack of this induction becomes evident in ADAM10 ${ }^{\triangle \mathrm{Alb}} \mathrm{ADAM} 17^{\triangle \mathrm{Alb}}$ after the challenge, while unchallenged animals resemble more ADAM10 $10^{\triangle \mathrm{Alb}}$ in respect to TNF RI shedding. Besides proteolytic shedding, soluble TNF RI can be released into serum by exosome-like vesicles [48]. It would be worth to investigate whether ADAM10 deficiency alters this export.

Altogether our data show, that hepatocyte-derived ADAM10 and ADAM17 contribute to the regulation of several distinct pathways, i.e. EGFR, HGF and TNF RI, during liver injury and regeneration. Potential therapies based on inhibition of ADAM10 and ADAM17 should carefully consider various roles of these proteases in different cell types.

\section{Materials And Methods}

\section{Mouse models}

Mouse lines ADAM10 flox on 129/C57BI/6 background [49] (kindly provided by Dr. Andreas Ludwig, RWTH Aachen University) and ADAM17 flox C57BI/6J [50] (kindly provided by Dr. Carl Blobel, HSS Research institute) were first backcrossed to $\mathrm{C} 57 \mathrm{BI} / 6 \mathrm{~N}$. N4 generations were crossed together to create ADAM10 flox ADAM17 flox line. All three floxed stains were crossed to line expressing Cre under the control of albumin promoter, Alb-Cre (B6.Cg-Tg(Alb-cre)21Mgn/J; The Jackson Laboratory). For liver 
challenges 9-14 weeks old males were used. Animals used in all experiments were homozygous for flox alleles and hemizygous for Cre recombinase and were termed ADAM10 $\triangle \mathrm{Alb}, \mathrm{ADAM} 17^{\triangle \mathrm{Alb}}, \mathrm{ADAM} 10^{\triangle \mathrm{Alb}}$ ADAM17 ${ }^{\triangle A l b}$. Littermates without Cre gene were used as control group (Ctrl) in each line.

Model of 2/3 partial hepatectomy was performed as described previously [51] with slight modifications. Briefly, mice were anesthetized with mixture of Zoletil (Virbac, Carros, France) and Rometar (Bioveta, Ivanovice, Czech Republic), frontal lobes and left lateral lobe are ligated with silk suture and subsequently removed. For sample harvesting mice were anesthetized at indicated time points after the surgery, blood was collected from orbital plexus, animals were subsequently euthanized by cervical dislocation and liver samples were taken from defined areas of remaining lobes - one piece was fixed for histological analysis (see below) and several smaller pieces were snap frozen and kept in $-80^{\circ} \mathrm{C}$ for RNA and protein isolation.

To induce fibrogenesis, mice were injected intraperitoneally with $1 \mu \mathrm{l} / \mathrm{g}$ of $\mathrm{CCl}_{4}$ (Sigma-Aldrich, Darmstadt, Germany) diluted 1:3 with olive oil twice a week for 4 weeks. Liver and blood samples were collected 48 hours after the last injection as described for partial hepatectomy model.

The animals were handled in accordance with the Guide for the Care and Use of Laboratory Animals, approved by the Animal Care and Use Committee of the Academy of Sciences of the Czech Republic. Mice were kept under standard laboratory conditions with free access to food and water.

\section{Primary hepatocyte isolation and treatment}

Hepatocytes were isolated by perfusion with collagenase I as described [52] [53]. Hepatocytes were plated on collagen I $(0.3 \mathrm{mg} / \mathrm{mL})$ and let to attach in DMEM containing $10 \%$ fetal bovine serum (FBS), $1 \%$ penicillin-streptomycin and $0.08 \mathrm{U} / \mathrm{mL}$ insulin (culture medium) for 24 hours before proceeding with experiments.

For shedding assay, cells were maintained in fresh culture medium for 6 hours. Harvested medium was cleared from debris by centrifugation $(10,000 \times \mathrm{g} / 10 \mathrm{~min})$ and analysed by ELISA. For HGF stimulation, cells were kept in culture media without FBS for 6 hours, then incubated with recombinant human HGF (20 ng/mL; R\&D Systems) in fresh medium for 30 minutes. After stimulation cells were immediately lysed with RIPA buffer (25 mM Tris, $150 \mathrm{mM} \mathrm{NaCl}, 10 \mathrm{mM}$ EDTA, 1 \% triton X-100, 1 \% DOC, 0.1 \% SDS, Complete ${ }^{\circledR}$ inhibitors (Roche), $1 \mathrm{mM}$ ortho-vanadate, $2.5 \mathrm{mM}$ sodium pyrophosphate, $1 \mathrm{mM} \beta$ glycerolphosphate).

For stimulation of primary hepatocytes with mouse serum, cells were kept in culture media without FBS for 6 hours, then treated for 30 min with DMEM medium supplemented with $10 \%$ of mouse sera and subsequently lysed with RIPA buffer. Samples were further processed for immunoblotting and ELISA.

\section{Histological Analysis}


Specimens were fixed in $4 \%$ buffered formaldehyde, embedded in paraffin, sectioned, and stained with hematoxylin and eosin (H\&E), Sirius Red [54], or processed for immunohistochemistry. To visualize proliferating cells, sections were stained with antibodies against ki-67 (Dako, Santa Clara, CA) and PCNA (Santa Cruz Biotechnology, Dallas, TX). For the analysis, 10 optical fields (original magnification $400 \mathrm{x}$ ) were taken per animal and positively stained nuclei were counted. Sirius red staining was quantified using ImageJ software [55].

\section{Analysis of Serum Parameters}

Collected blood was allowed to cloth for 1 hour in $37^{\circ} \mathrm{C}$, spun down $(10,000 \mathrm{~g} / 20 \mathrm{~min})$, and cleared serum was kept frozen before further analysis. Serum levels of IL-6, KC, soluble c-Met and soluble TNFreceptor I were assayed by ELISA using antibodies from R\&D Systems (Minneapolis, MN). Activities of alanine aminotransferase (ALT), aspartate aminotransferase (AST) and alkaline phosphatase (ALP) were assessed using kits from Roche Diagnostics (Basel, Switzerland).

\section{Immunoblotting}

Frozen pieces of liver tissues were homogenized in RIPA buffer using TissueLyzer II (Qiagen, Hilden, Germany). Equal amounts of protein as assessed by BCA assay were resolved on SDS polyacrylamide gels and transferred to nitrocellulose membranes; proteins were detected using antibodies against phospho c-Met, phospho-AKT, AKT, phospho-EGFR, EGFR (all Cell Signaling Technology, Danvers, MA) and GAPDH (Sigma-Aldrich, Darmstadt, Germany) as loading control. Densitometric analysis was performed by AIDA Image Analyzer software (Raytest).

\section{Quantitative Reverse-Transcriptase Polymerase Chain Reaction (qRT-PCR)}

Total RNA was isolated from piece of snap frozen mouse liver tissue by TRI Reagent $\circledast$ (Sigma-Aldrich). mRNA was transcribed using polyT primers and MLV-RT (Promega, Fitchburg, WI). For RT reaction, SYBR ${ }^{\circledR}$ Green JumpStart ${ }^{\text {TM }}$ Taq ReadyMix ${ }^{\text {TM }}$ (Sigma-Aldrich) was used. Primers for amplification of mRNA were used as follows: Adam10 (5'-GCTGGGAGGTCAGTATGGAA-3', 5'-TGGTCCTCATGTGAGACTGC-3'), Adam17 (5'-CCACCACCACGACTCTCAAG-3', 5'-CAGTCTGCGACACACTTAGAAAC-3'), Tnfrl (5'-

TAACTGCCATGCAGGGTTCT-3', 5'-CTGGGGGTTTGTGACATTTG-3'). Data were normalized to Gapdh (5'CGTCCCGTAGACAAAATGGT-3', 5'-TTGATGGCAACAATCTCCAC-3') and Hprt (5'-

TCCTCCTCAGACCGCTTTT-3', 5'-CCTGGTTCATCATCGCTAAT-3').

\section{Statistical analysis}

Data are shown as mean \pm SEM. For analysis we used Student's t-test (GraphPad Prism). P values were considered significant under $0.05:{ }^{*} p<0.05,{ }^{* \star} p<0.01$, ${ }^{\star \star *} p<0.001 .4-10$ mice from each line per time point were used. Data from primary hepatocytes were collected from three independent isolations. 


\section{Declarations}

\section{Acknowledgement}

We thank Attila Juhasz and Marketa Pickova for excellent technical support and Miles Raishbrook for proof-reading the manuscript. The study was supported by RVO 68378050 by Academy of Sciences of the Czech Republic and by LM2015040 and LM2018126 (Czech Centre for Phenogenomics),

CZ.1.05/2.1.00/19.0395 ('Higher quality and capacity for transgenic models'), CZ.1.05/1.1.00/02.0109 (BIOCEV - Biotechnology and Biomedicine Centre of the Academy of Sciences and Charles University), LQ1604 (National Sustainability Program II project BIOCEV-FAR) funded by the Ministry of Education, Youth and Sports and the European Regional Development Fund.

\section{AUTHOR CONTRIBUTIONS}

O.Z. designed and performed experiments, prepared figures and manuscript, K.C. designed and performed experiments, L.S. isolated primary hepatocytes and helped with mouse experiments, M.G. designed experiments and participated in manuscript preparation, M.J. discussed results and designed experiments, P.K. was involved in manuscript preparation, R.S. was involved in planning experiments, discussion and manuscript preparation.

\section{Competing Interests Statements}

The authors declare no competing interests.

\section{References}

1. Michalopoulos, G. K. Liver regeneration after partial hepatectomy: critical analysis of mechanistic dilemmas. Am J Patho/ 176, 2-13, doi:10.2353/ajpath.2010.090675 (2010).

2. Taub, R. Liver regeneration: from myth to mechanism. Nat Rev Mol Cell Bio/ 5, 836-847, doi:10.1038/nrm1489 (2004).

3. Iwai, M., Cui, T. X., Kitamura, H., Saito, M. \& Shimazu, T. Increased secretion of tumour necrosis factor and interleukin 6 from isolated, perfused liver of rats after partial hepatectomy. Cytokine 13, 60-64, doi:10.1006/cyto.2000.0797 (2001).

4. Selzner, N. et al. ICAM-1 triggers liver regeneration through leukocyte recruitment and Kupffer celldependent release of TNF-alpha/IL-6 in mice. Gastroenterology 124, 692-700, doi:10.1053/gast.2003.50098 (2003).

5. Natarajan, A., Wagner, B. \& Sibilia, M. The EGF receptor is required for efficient liver regeneration. Proc Natl Acad Sci U S A 104, 17081-17086, doi:10.1073/pnas.0704126104 (2007).

6. Collin de L'hortet, A., Gilgenkrantz, H. \& Guidotti, J. E. EGFR: A Master Piece in G1/S Phase Transition of Liver Regeneration. Int J Hepatol 2012, 476910, doi:10.1155/2012/476910 (2012). 
7. Seki, E. et al. Demonstration of cooperative contribution of MET- and EGFR-mediated STAT3 phosphorylation to liver regeneration by exogenous suppressor of cytokine signalings. J Hepatol 48, 237-245, doi:10.1016/j.jhep.2007.08.020 (2008).

8. Zhang, X. J. et al. Angiocrine Hepatocyte Growth Factor Signaling Controls Physiological Organ and Body Size and Dynamic Hepatocyte Proliferation to Prevent Liver Damage during Regeneration. Am J Pathol, doi:10.1016/j.ajpath.2019.10.009 (2019).

9. Tsai, M. T. et al. Regulation of HGF-induced hepatocyte proliferation by the small GTPase Arf6 through the PIP2-producing enzyme PIP5K1A. Sci Rep 7, 9438, doi:10.1038/s41598-017-09633-z (2017).

10. Tsagianni, A. et al. Combined Systemic Disruption of MET and Epidermal Growth Factor Receptor Signaling Causes Liver Failure in Normal Mice. Am J Pathol 188, 2223-2235, doi:10.1016/j.ajpath.2018.06.009 (2018).

11. Paranjpe, S. et al. Combined systemic elimination of MET and epidermal growth factor receptor signaling completely abolishes liver regeneration and leads to liver decompensation. Hepatology 64, 1711-1724, doi:10.1002/hep.28721 (2016).

12. Singh, B., Carpenter, G. \& Coffey, R. J. EGF receptor ligands: recent advances. F1000Res 5, doi:10.12688/f1000research.9025.1 (2016).

13. Mead, J. E. \& Fausto, N. Transforming growth factor alpha may be a physiological regulator of liver regeneration by means of an autocrine mechanism. Proc Natl Acad Sci U S A 86, 1558-1562, doi:10.1073/pnas.86.5.1558 (1989).

14. Kiso, S. et al. Role of heparin-binding epidermal growth factor-like growth factor as a hepatotrophic factor in rat liver regeneration after partial hepatectomy. Hepatology 22, 1584-1590 (1995).

15. Skov Olsen, P. et al. Influence of epidermal growth factor on liver regeneration after partial hepatectomy in rats. Hepatology 8, 992-996, doi:10.1002/hep.1840080503 (1988).

16. Mitchell, C. et al. Heparin-binding epidermal growth factor-like growth factor links hepatocyte priming with cell cycle progression during liver regeneration. J Biol Chem 280, 2562-2568, doi:10.1074/jbc.M412372200 (2005).

17. Berasain, C. et al. Amphiregulin: an early trigger of liver regeneration in mice. Gastroenterology 128, 424-432 (2005).

18. Naldini, L. et al. Hepatocyte growth factor (HGF) stimulates the tyrosine kinase activity of the receptor encoded by the proto-oncogene c-MET. Oncogene 6, 501-504 (1991).

19. Skrtic, S. et al. Hepatocyte-stimulated expression of hepatocyte growth factor (HGF) in cultured rat hepatic stellate cells. J Hepato/30, 115-124, doi:10.1016/s0168-8278(99)80015-4 (1999).

20. Matthews, A. L., Noy, P. J., Reyat, J. S. \& Tomlinson, M. G. Regulation of A disintegrin and metalloproteinase (ADAM) family sheddases ADAM10 and ADAM17: The emerging role of tetraspanins and rhomboids. Platelets 28, 333-341, doi:10.1080/09537104.2016.1184751 (2017).

21. Hartmann, D. et al. The disintegrin/metalloprotease ADAM 10 is essential for Notch signalling but not for alpha-secretase activity in fibroblasts. Hum Mol Genet 11, 2615-2624 (2002). 
22. Peschon, J. J. et al. An essential role for ectodomain shedding in mammalian development. Science 282, 1281-1284 (1998).

23. Muller, M. et al. A disintegrin and metalloprotease 10 (ADAM10) is a central regulator of murine liver tissue homeostasis. Oncotarget 7, 17431-17441, doi:10.18632/oncotarget.7836 (2016).

24. Reddy, P. et al. Functional analysis of the domain structure of tumor necrosis factor-alpha converting enzyme. J Biol Chem 275, 14608-14614, doi:10.1074/jbc.275.19.14608 (2000).

25. McMahan, R. S., Riehle, K. J., Fausto, N. \& Campbell, J. S. A disintegrin and metalloproteinase 17 regulates TNF and TNFR1 levels in inflammation and liver regeneration in mice. Am J Physiol Gastrointest Liver Physio/ 305, G25-34, doi:10.1152/ajpgi.00326.2012 (2013).

26. Casagrande, V. et al. Hepatocyte specific TIMP3 expression prevents diet dependent fatty liver disease and hepatocellular carcinoma. Sci Rep 7, 6747, doi:10.1038/s41598-017-06439-x (2017).

27. Sahin, U. et al. Distinct roles for ADAM10 and ADAM17 in ectodomain shedding of six EGFR ligands. J Cell Biol 164, 769-779, doi:10.1083/jcb.200307137 (2004).

28. Asakura, M. et al. Cardiac hypertrophy is inhibited by antagonism of ADAM12 processing of HB-EGF: metalloproteinase inhibitors as a new therapy. Nat Med 8, 35-40, doi:10.1038/nm0102-35 (2002).

29. Yang, Y. et al. Self-control of HGF regulation on human trophoblast cell invasion via enhancing c-Met receptor shedding by ADAM10 and ADAM17. J Clin Endocrinol Metab 97, E1390-1401, doi:10.1210/jc.2012-1150 (2012).

30. Chalupsky, K. et al. ADAM10/17-dependent release of soluble c-Met correlates with hepatocellular damage. Folia biologica 59, 76-86 (2013).

31. Jackson, L. N. et al. PI3K/Akt activation is critical for early hepatic regeneration after partial hepatectomy. Am J Physiol Gastrointest Liver Physio/ 294, G1401-1410, doi:10.1152/ajpgi.00062.2008 (2008).

32. Ijiri, Y. et al. Chronological changes in circulating levels of soluble tumor necrosis factor receptors 1 and 2 in rats with carbon tetrachloride-induced liver injury. Toxicology 316, 55-60, doi:10.1016/j.tox.2013.12.004 (2014).

33. Sundaram, B. et al. iRhom2 inhibits bile duct obstruction-induced liver fibrosis. Sci Signal 12, doi:10.1126/scisignal.aax1194 (2019).

34. Deng, M., Loughran, P. A., Zhang, L., Scott, M. J. \& Billiar, T. R. Shedding of the tumor necrosis factor (TNF) receptor from the surface of hepatocytes during sepsis limits inflammation through cGMP signaling. Sci Signa/ 8, ra11, doi:10.1126/scisignal.2005548 (2015).

35. Xanthoulea, S. et al. Tumor necrosis factor (TNF) receptor shedding controls thresholds of innate immune activation that balance opposing TNF functions in infectious and inflammatory diseases. $J$ Exp Med 200, 367-376, doi:10.1084/jem.20040435 (2004).

36. Scheving, L. A., Zhang, X., Stevenson, M. C., Threadgill, D. W. \& Russell, W. E. Loss of hepatocyte EGFR has no effect alone but exacerbates carbon tetrachloride-induced liver injury and impairs regeneration in hepatocyte Met-deficient mice. Am J Physiol Gastrointest Liver Physio/ 308, G364377, doi:10.1152/ajpgi.00364.2014 (2015). 
37. Wilson, K. J. et al. EGFR ligands exhibit functional differences in models of paracrine and autocrine signaling. Growth Factors 30, 107-116, doi:10.3109/08977194.2011.649918 (2012).

38. Pardo-Saganta, A. et al. The epidermal growth factor receptor ligand amphiregulin is a negative regulator of hepatic acute-phase gene expression. J Hepato/ 51, 1010-1020, doi:10.1016/j.jhep.2009.06.030 (2009).

39. Olsen, P. S., Poulsen, S. S. \& Kirkegaard, P. Adrenergic effects on secretion of epidermal growth factor from Brunner's glands. Gut 26, 920-927 (1985).

40. Horiuchi, K. et al. Substrate selectivity of epidermal growth factor-receptor ligand sheddases and their regulation by phorbol esters and calcium influx. Mol Biol Cell 18, 176-188, doi:10.1091/mbc.E06-01-0014 (2007).

41. Borowiak, M. et al. Met provides essential signals for liver regeneration. Proc Natl Acad Sci U S A 101, 10608-10613, doi:10.1073/pnas.0403412101 (2004).

42. Aleksunes, L. M., Scheffer, G. L., Jakowski, A. B., Pruimboom-Brees, I. M. \& Manautou, J. E. Coordinated expression of multidrug resistance-associated proteins (Mrps) in mouse liver during toxicant-induced injury. Toxicol Sci 89, 370-379, doi:10.1093/toxsci/kfi332 (2006).

43. He, Y. et al. Interaction of CD44 and hyaluronic acid enhances biliary epithelial proliferation in cholestatic livers. Am J Physiol Gastrointest Liver Physiol 295, G305-312, doi:10.1152/ajpgi.90229.2008 (2008).

44. Anderegg, U. et al. ADAM10 is the constitutive functional sheddase of CD44 in human melanoma cells. J Invest Dermato/ 129, 1471-1482, doi:10.1038/jid.2008.323 (2009).

45. Buryova, H. et al. Liver protective effect of ursodeoxycholic acid includes regulation of ADAM17 activity. BMC Gastroentero/ 13, 155, doi:10.1186/1471-230X-13-155 (2013).

46. Shibata, H. et al. The therapeutic effect of TNFR1-selective antagonistic mutant TNF-alpha in murine hepatitis models. Cytokine 44, 229-233, doi:10.1016/j.cyto.2008.07.003 (2008).

47. Yamada, Y. \& Fausto, N. Deficient liver regeneration after carbon tetrachloride injury in mice lacking type 1 but not type 2 tumor necrosis factor receptor. Am J Pathol 152, 1577-1589 (1998).

48. Berkhout, L. et al. Deletion of tumour necrosis factor alpha receptor 1 elicits an increased TH17 immune response in the chronically inflamed liver. Sci Rep 9, 4232, doi:10.1038/s41598-019-40324-z (2019).

49. Hawari, F. I. et al. Release of full-length 55-kDa TNF receptor 1 in exosome-like vesicles: a mechanism for generation of soluble cytokine receptors. Proc Natl Acad Sci U S A 101, 1297-1302, doi:10.1073/pnas.0307981100 (2004).

50. Jorissen, E. et al. The disintegrin/metalloproteinase ADAM10 is essential for the establishment of the brain cortex. J Neurosci 30, 4833-4844, doi:10.1523/JNEUROSCI.5221-09.2010 (2010).

51. Horiuchi, K. et al. Cutting edge: TNF-alpha-converting enzyme (TACE/ADAM17) inactivation in mouse myeloid cells prevents lethality from endotoxin shock. J Immunol 179, 2686-2689, doi:10.4049/jimmunol.179.5.2686 (2007). 
52. Mitchell, C. \& Willenbring, H. A reproducible and well-tolerated method for $2 / 3$ partial hepatectomy in mice. Nat Protoc 3, 1167-1170, doi:10.1038/nprot.2008.80 (2008).

53. Moldeus, P., Hogberg, J. \& Orrenius, S. Isolation and use of liver cells. Methods Enzymo/ 52, 60-71 (1978).

54. Korelova, K., Jirouskova, M., Sarnova, L. \& Gregor, M. Isolation and 3D Collagen Sandwich Culture of Primary Mouse Hepatocytes to Study the Role of Cytoskeleton in Bile Canalicular Formation In Vitro. J Vis Exp, doi:10.3791/60507 (2019).

55. Jirouskova, M. et al. Hepatoprotective effect of MMP-19 deficiency in a mouse model of chronic liver fibrosis. PLoS One 7, e46271, doi:10.1371/journal.pone.0046271 (2012).

56. Schindelin, J. et al. Fiji: an open-source platform for biological-image analysis. Nat Methods 9, 676682, doi:10.1038/nmeth.2019 (2012).

\section{Figures}


Fig.1

a
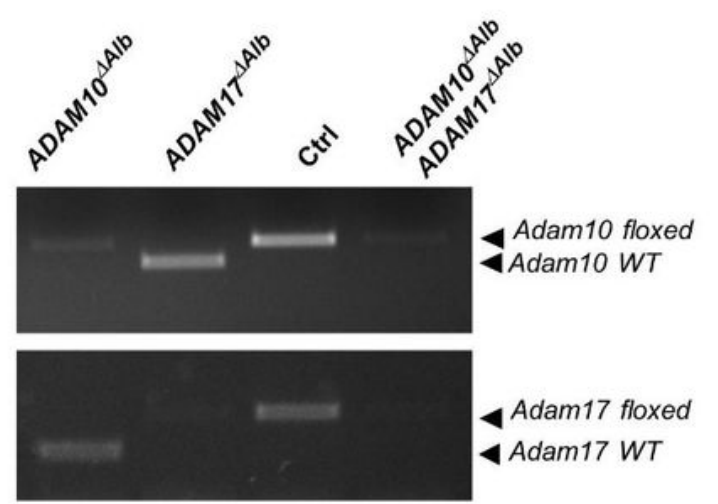

Adam17 floxed 4 Adam17 WT

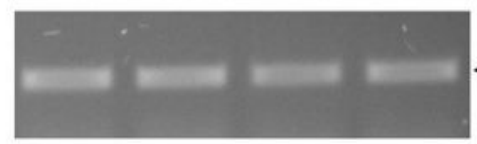

Gapdh b
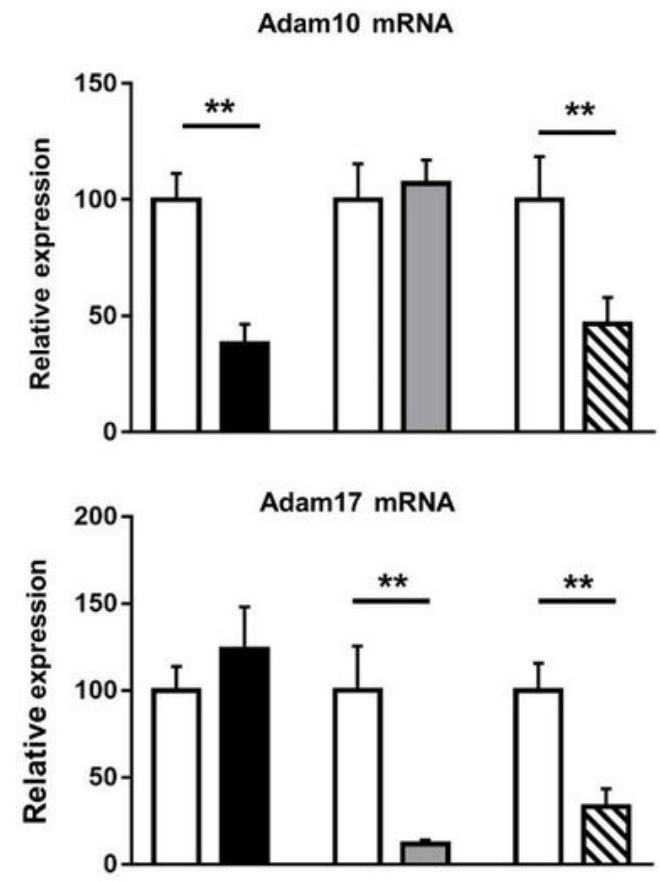

C
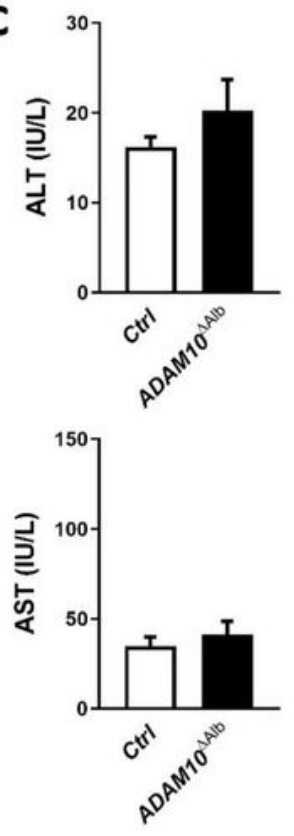
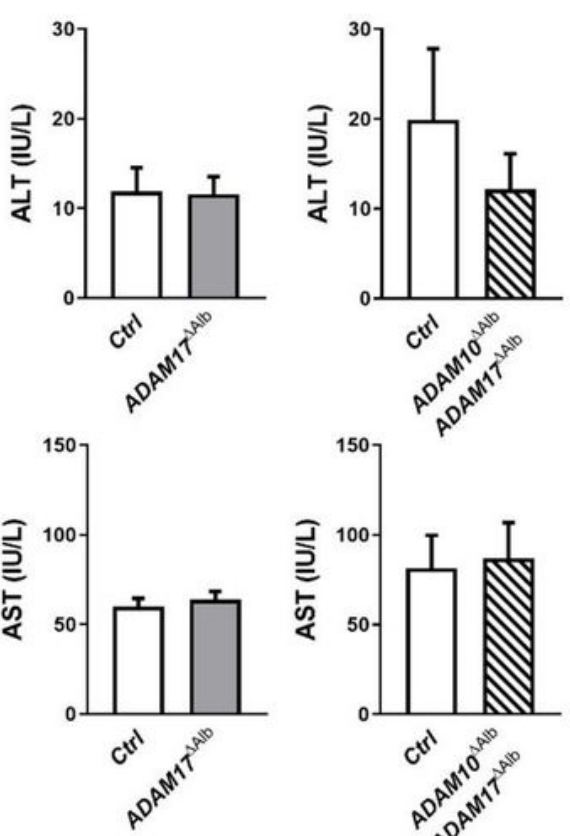

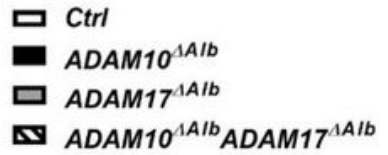

\section{Figure 1}

Alb-Cre mediated deletion of ADAM10 and ADAM17 genes (a) Semiquantitative PCR showed almost complete elimination of floxed exons in genomic DNA of primary hepatocytes isolated from ADAM10 $\triangle$ Alb, ADAM17 $\triangle$ Alb and ADAM10 $\triangle$ AlbADAM17 $\triangle$ Alb mice. Cre negative littermates from ADAM10 $\triangle$ AlbADAM17 $\triangle$ Alb were used as controls. (b) Quantitative RT-PCR showed significant reduction of Adam10 and Adam17 mRNA levels in liver tissue of ADAM10 AAlb, ADAM17 $\triangle$ Alb and 
ADAM10 $\triangle$ AlbADAM17 $\triangle$ Alb mice. Expression was normalized to Gapdh. $(n=5$ per group, mean \pm SEM, $\star \star p<0.01)$ (c) ALT and AST levels in serum from unchallenged 32 weeks old mice shows no hepatocellular damage in all studied lines. $(n=4-10$ per group, mean \pm SEM)

Fig.2

a
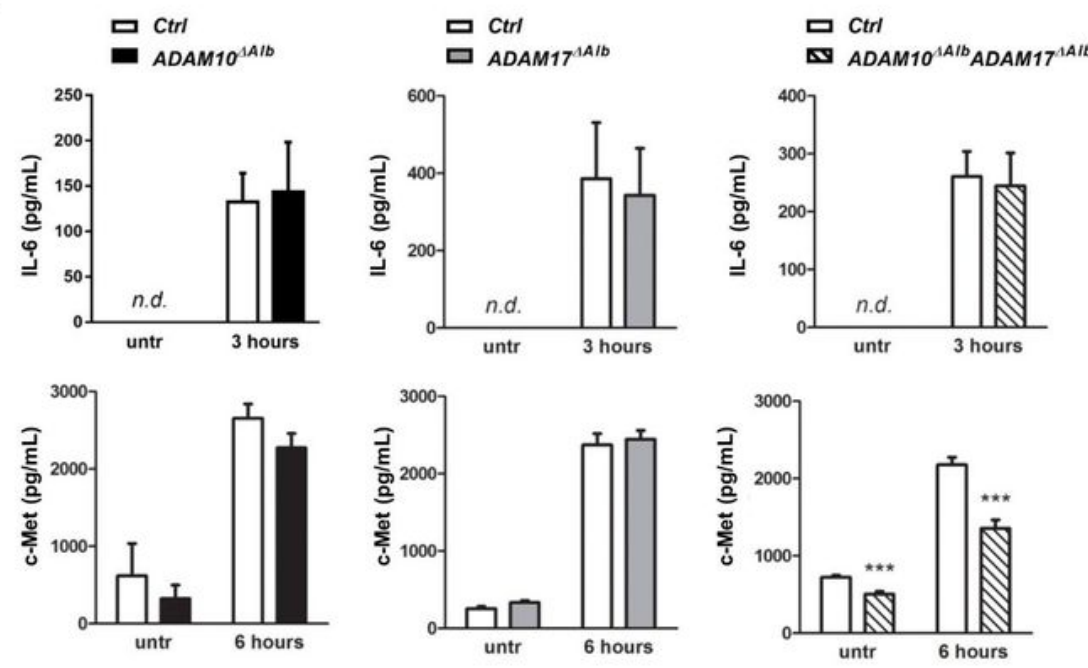

b

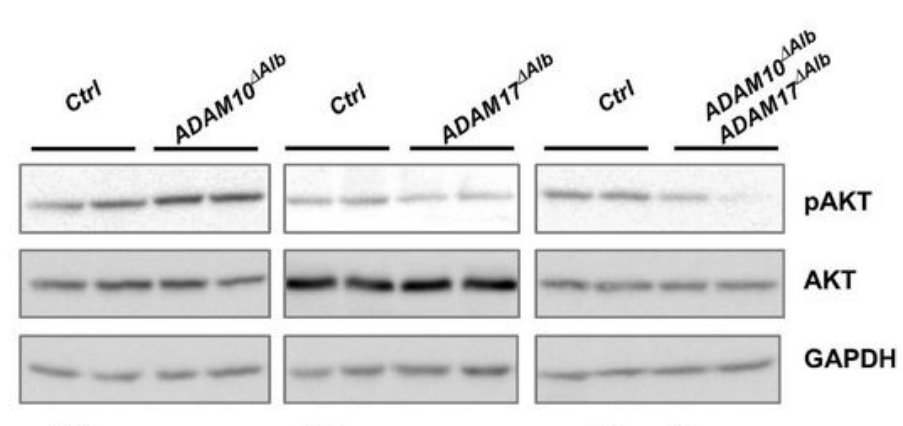

C

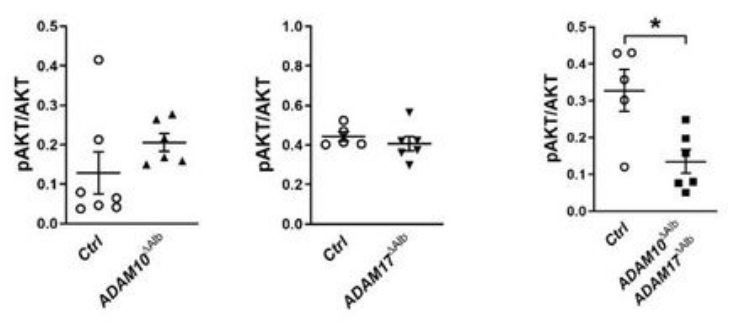

d

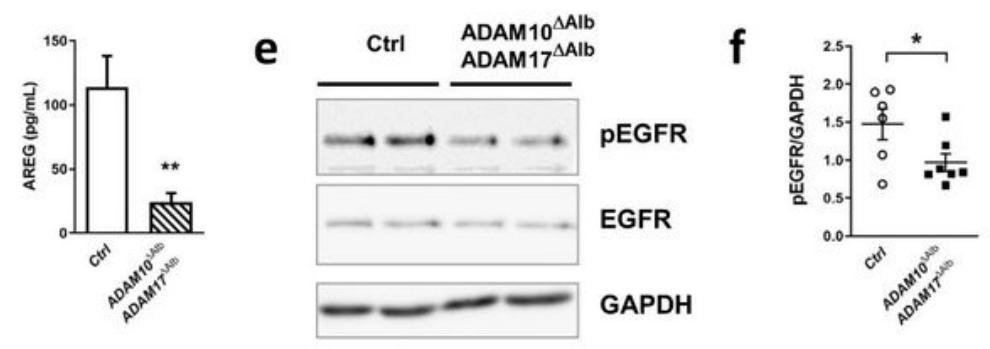

Figure 2

Analysis of prosurvival pathways in priming phase of liver regeneration (a) Elevated levels of IL- 6 and CMet determined by ELISA in serum from mice 3 and 6 hours post hepatectomy were observed in all 
studied groups. Shedding of c-Met was significantly ablated in ADAM10 $\triangle$ AlbADAM17 $\triangle$ Alb versus Ctrl littermates. ( $n=6-10$ mice per group in each time point, mean \pm SEM, $* \star \star p<0.001)$ (b) phospho-AKT (pAKT) and AKT in liver lysates from animals 6 hours post hepatectomy was evaluated by immunoblotting. ADAM10 $\triangle$ AlbADAM17 $\triangle$ Alb exhibited significantly lesser extent of AKT phosphorylation than Ctrl. (c) Densitometric analysis of immunoblot from (b) $\left.{ }^{*} \mathrm{k}<0.05\right)$. (d) Analysis of culture media by ELISA determined reduced shedding of AREG from cell surface of ADAM10 $\triangle$ AlbADAM17 $\triangle$ Alb primary hepatocytes compared to Ctrl. (e) Ctrl primary hepatocytes were stimulated for 30 min with sera taken from mice 6 hours post hepatectomy. Cell lysates analysed by immunoblotting showed that mouse serum from ADAM10 $\triangle$ AlbADAM17 $\triangle$ Alb mice has reduced ability to induce phosphorylation of EGFR compared to Ctrl serum. ( $\left.{ }^{\star \star} p<0.01\right)$ (f) Densitometry of immunoblot from (e) $\left({ }^{\star} p<0.05\right)$. 
Fig.3

a

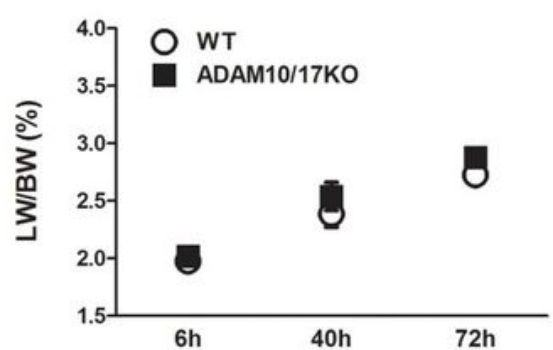

C

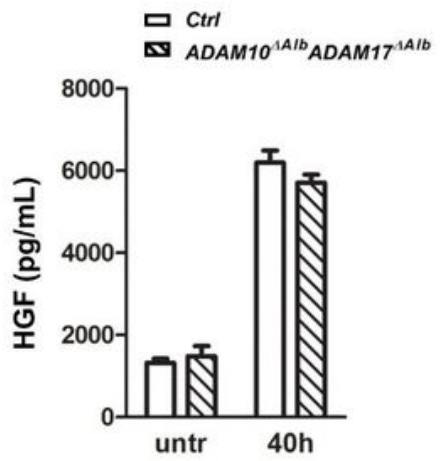

e

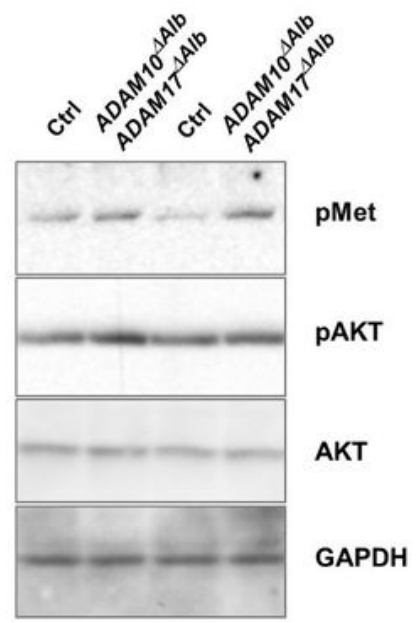

b

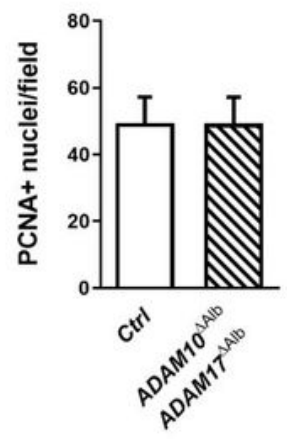

d

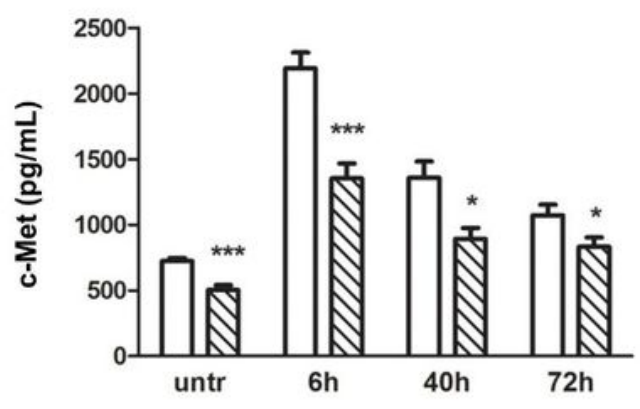

f
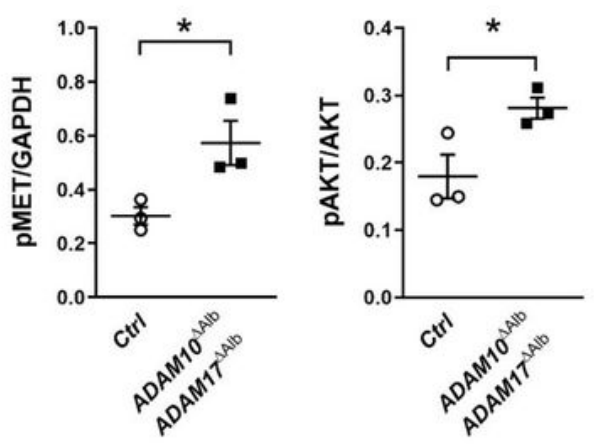

\section{Figure 3}

HGF signalling in ADAM10 $\triangle$ AlbADAM17 $\triangle$ Alb after hepatectomy. (a) Gain of relative liver weight in ADAM10 $\triangle$ AlbADAM17 $\triangle$ Alb and Ctrl was monitored before and 6, 40 and 72 hours post hepatectomy. No differences between groups were observed in liver weight to body weight ratio (LW/BW). ( $n=8-10$ mice from each line per time point) (b) Immunohistochemical staining of liver sections revealed no difference in number of PCNA positive (PCNA+) between ADAM10 $\triangle$ AlbADAM17 $\triangle$ Alb and Ctrl littermates 40 hours 
post hepatectomy. (c) Serum from mice 40 post hepatectomy exhibits elevated levels of HGF in both ADAM10 $\triangle$ AlbADAM17 $\triangle$ Alb and Ctrl animals. (d) Levels of shed c-Met into serum were lower in ADAM10 $\triangle$ AlbADAM17 $\triangle$ Alb in all studied time points post hepatectomy. $(n=5-9$, mean $\pm S E M, * p<0.05$, $\left.{ }^{* \star *} \mathrm{p}<0.001\right)(\mathrm{e})$ Phosphorylation of $\mathrm{c}-$ Met receptor (pMet) and AKT in primary hepatocytes after $30 \mathrm{~min}$ HGF treatment was analysed in lysates by immunoblotting. ADAM10 $\triangle$ AlbADAM17 $\triangle$ Alb show higher phosphorylation of cMet and AKT than Ctrl. (f) Densitometric evaluation of immunoblots from (e). Representative of three independent experiments shown.

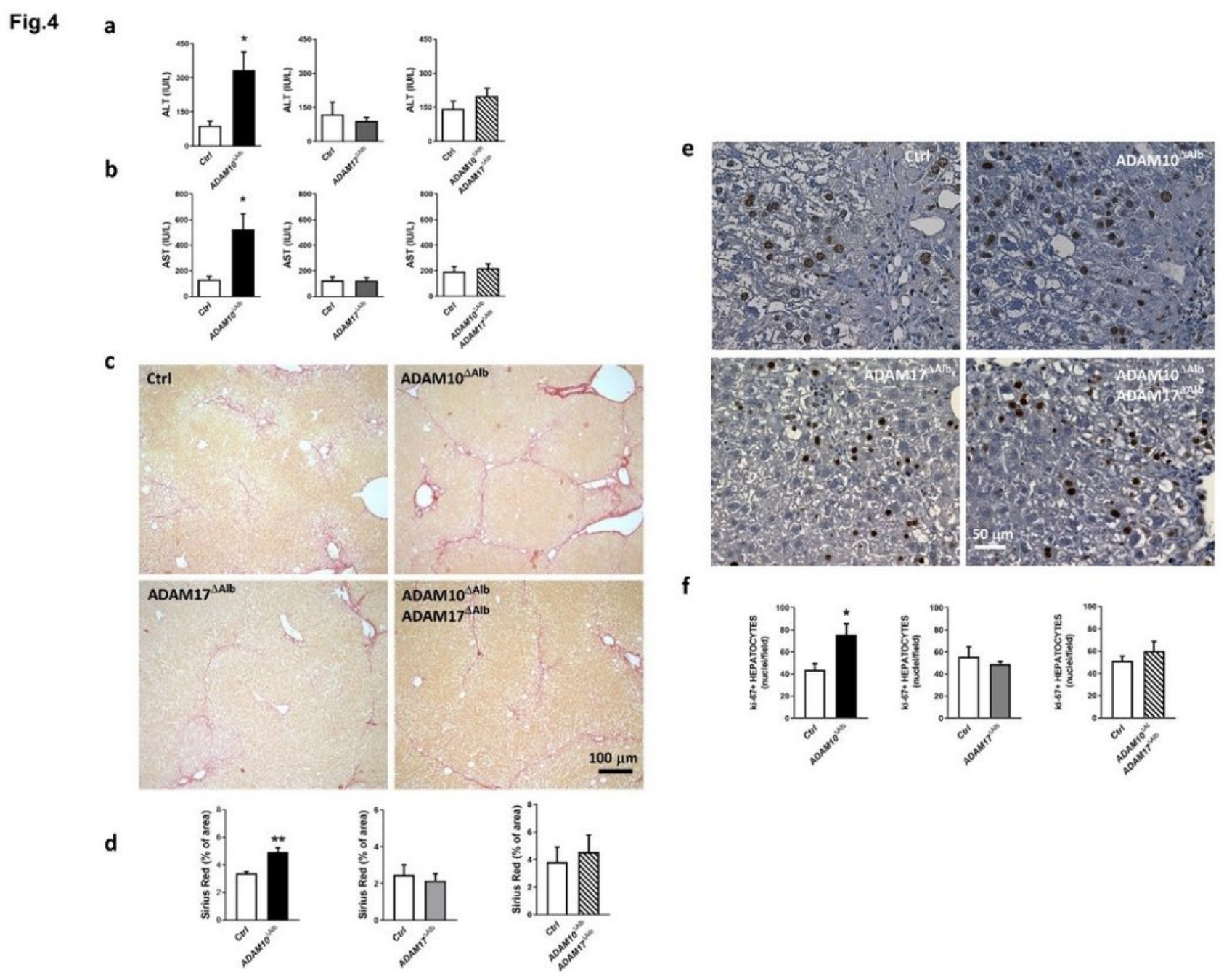

\section{Figure 4}

Model of fibrogenesis induced by CCl4 intoxication. (a), (b) Serum levels of ALT and AST from mice after 4-weeks treatment with $\mathrm{CCl} 4$ show highest hepatocyte damage in ADAM10 $\triangle$ Alb mice. ( $n=4-10$ mice per group, ${ }^{*}<0.05$ ) (c) Sirius red staining shows higher deposition of fibrotic collagens in ADAM10 $\triangle$ Alb than other studied groups. (d) Quantitative analysis of Sirius red -positive (Sirius Red + ) area. $(n=3-7$, mean \pm SEM, $\left.{ }^{* *} \mathrm{p}<0.01\right)$ (e) Ki-67 staining shows than number of proliferating hepatocytes was highest in ADAM10 $\triangle$ Alb after 4-weeks treatment with CCl4. (f) Quantitative analysis of ki-67 staining. Only nuclei corresponding to hepatocytes (identified by shape) were counted. $(n=4-7$, mean $\pm S E M, * p<0.05$ ) 
Fig.5

a
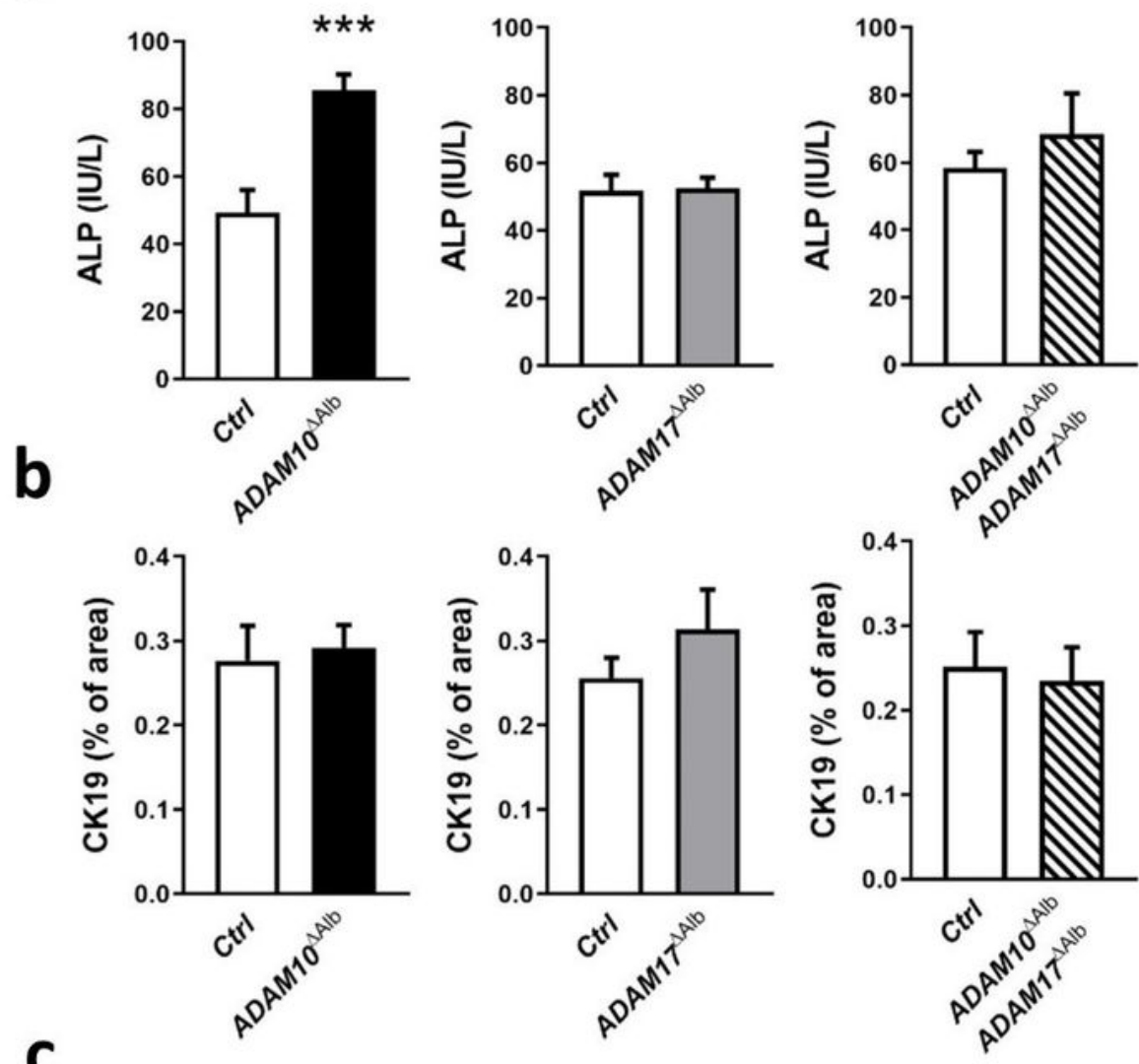

C

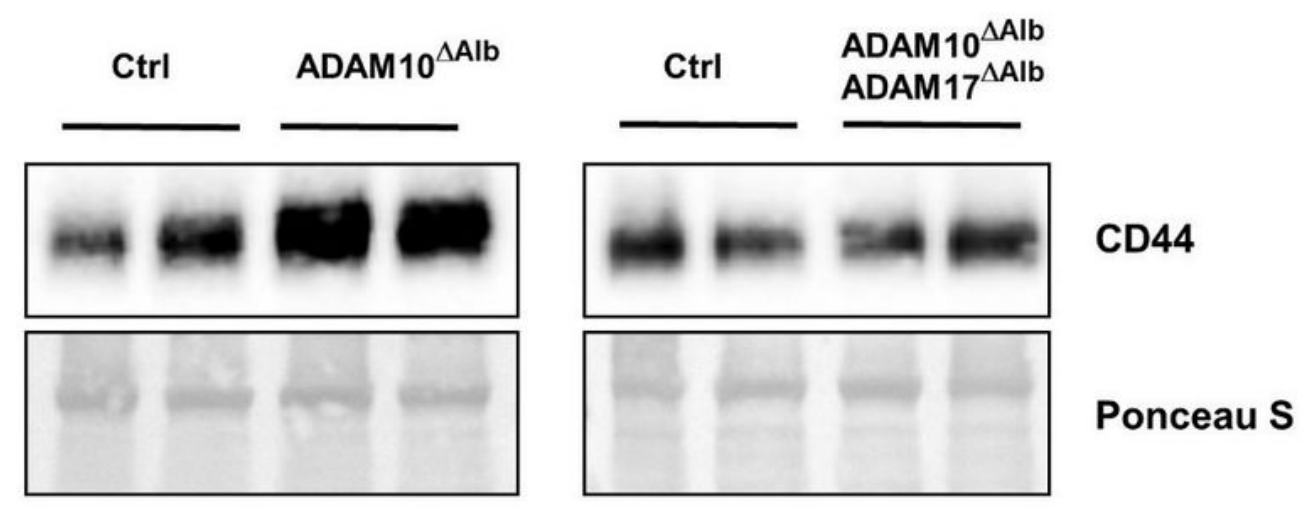

Figure 5

Effect of CCl4 intoxication on biliary epithelial cells. (a) Serum levels of ALP were elevated in ADAM10 $\triangle$ Alb mice in comparison to other groups after 4-weeks treatment with $\mathrm{CCl}$. (b) Immunohistochemical analysis of CK19 in liver sections did not reveal any differences between studied groups. ( $n=4-7$ mice per group, mean \pm SEM) (c) Immunoblotting showed higher levels of CD44 in liver tissue of ADAM10 $\triangle$ Alb mice after 4-weeks treatment with $\mathrm{CCl} 4$ ( $\mathrm{n}=4$ mice per group). 
Fig.6

a

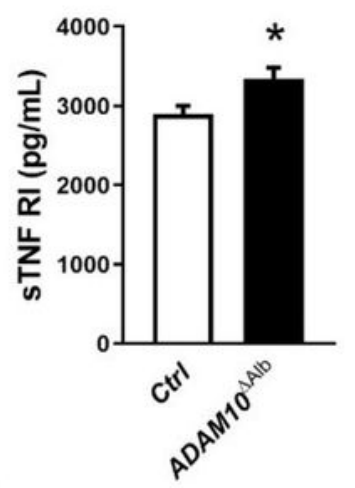

C

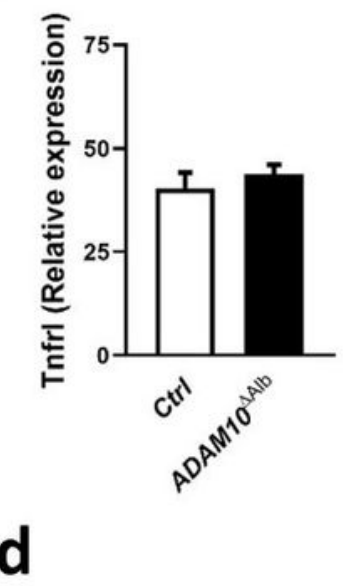

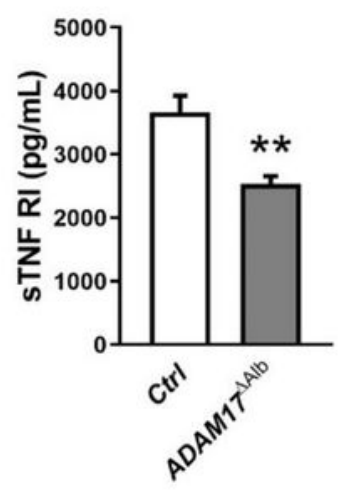
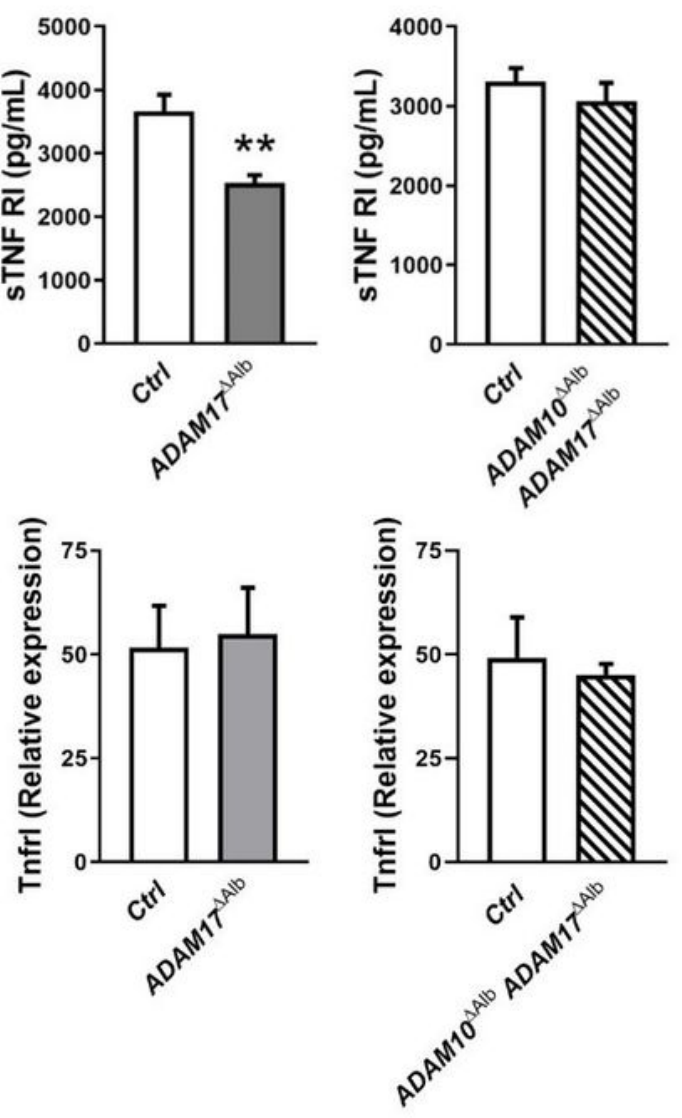

b

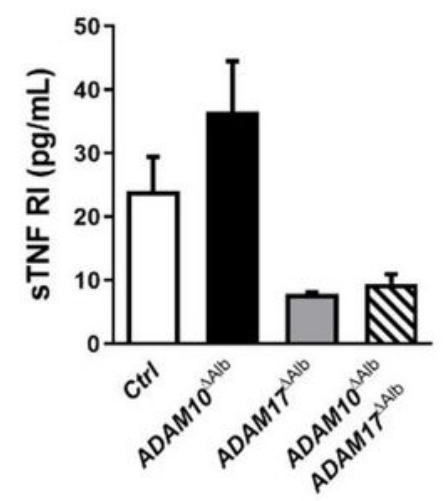

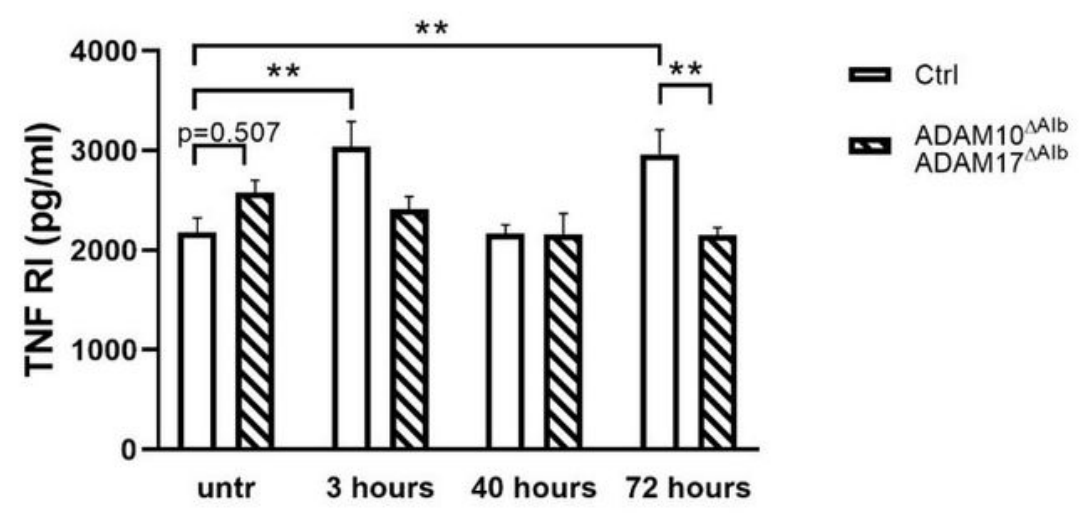

Figure 6

TNF RI shedding in CCI4 intoxication. (a) Higher shedding of TNF RI into serum was observed 4 weeks after $\mathrm{CCl} 4$ treatment in all studied groups as determined by ELISA. ADAM17 $\triangle$ Alb mice had lower levels of soluble TNF RI (sTNF RI) than Ctrl littermates, while in ADAM10 $\triangle$ Alb mice sTNF RI levels were elevated ( $\mathrm{n}$ = 4-10 per group, mean $\pm S E M,{ }^{*} p<0.05$, ${ }^{*} p<0.01$ ). (b) Levels of shed $s T N F$ RI from primary hepatocytes into culture medium after 6 hours of cultivation. ( $n=3$ per group, mean \pm SEM). (c) Expression of Tnfrl 
transcript in livers after CCL4 treatmnet was determined by q-PCR. Values were normalized to Gapdh and Hprt. ( $n=5$ per group, mean \pm SEM). (d) Serum levels of sTNF RI were determined by ELISA. Untreated ADAM10 $\triangle$ AlbADAM17 $\triangle$ Alb exhibited higher levels of sTNF RI in serum. After partial hepatectomy, levels of sTNF RI increased in Ctrl littermates, but not in the ADAM10 $A$ AlbADAM17 $\triangle$ Alb $(n=6-10$ per group, mean \pm SEM, $\left.{ }^{* *} p<0.01\right)$.

\section{Supplementary Files}

This is a list of supplementary files associated with this preprint. Click to download.

- 210205supplements.pdf 